Pacific Journal of Mathematics

TENSIONS OF HAUSDORFF SPACES

Ray PoRter and R. Grant Wood 


\title{
EXTENSIONS OF HAUSDORFF SPACES
}

\author{
JACK R. Porter AND R. GRANT WoOdS
}

A topological property $\mathscr{P}$ is called a Hausdorff extension property if each Hausdorff space $X$ can be densely embedded in a space $\kappa_{\mathscr{O}} X$ such that (1) $\kappa_{\mathscr{O}} X$ is a Hausdorff space with property $\mathscr{P},(2)$ if $Y$ is a Hausdorff extension of $X$ with $\mathscr{P}$, then there is a continuous function $f=\kappa_{\mathscr{P}} X \rightarrow Y$ such that $f(x)=x$ for each $x \in X$, and (3) if $X$ is $H$-closed, then $\kappa_{\mathscr{S}} X=X$. Both necessary conditions and sufficient conditions are given to characterize Hausdorff extension properties. Certain types of Hausdorff extension properties are shown to divide into classes such that each class has a largest member. In the latter part of the paper, for a Hausdorff extension property $\mathscr{P}$ satisfying one additional property, the lattice of $\mathscr{P}$-extensions of a fixed space $X$ is related to $\kappa_{\mathscr{P}} X \backslash X$ with a modified topology; this yields a theorem parallel to a similar result for the lattice of Hausdorff compactifications of a locally compact space $X$ and $\beta X \backslash X$ obtained by Magill.

1. Introduction. A topological property $\mathscr{P}$ is called a Tychonoff extension property if each Tychonoff space $X$ can be embedded as a dense subspace of a space $\beta_{\mathscr{O}} X$ with the following properties:

(1) $\beta_{\mathscr{P}} X$ is a Tychonoff space with $\mathscr{P}$.

(2) A continuous function $f: X \rightarrow Y$ between Tychonoff spaces $X$ and $Y$ has a continuous extension $F: \beta_{\mathscr{P}} X \rightarrow \beta_{\mathscr{P}} Y$.

(3) If $X$ has $\mathscr{P}$, then $X=\beta_{\mathscr{S}} X$.

In 1967 Herrlich and van der Slot [6] obtained the following characterization of those topological properties $\mathscr{P}$ that are Tychonoff extension properties:

(a) All compact Hausdorff spaces have $\mathscr{P}$.

(b) Closed subspaces of spaces with $\mathscr{P}$ have $\mathscr{P}$.

(c) Products of spaces with $\mathscr{P}$ have $\mathscr{P}$.

In 1975 the second-named author [20] developed systematic means of defining and classifying Tychonoff extension properties.

In this paper the corresponding problem concerning extensions of Hausdorff spaces is systematically studied. The two major models of Hausdorff extensions are the Katetov extensions [9] which generate the class of $H$-closed spaces and thd Liu-Strecker extensions [10] which generate the class of almost realcompact spaces. Based on these models, it is straightforward to find the common characteristics that Hausdorff extension properties should have; (1) and (2) are modified by replacing "Tychonoff" by "Hausdorff", (2) is modified by 
replacing "continuous function" by "dense embedding", and (3) is modified by replacing "compact Hausdorff" by " $H$-closed". Topological properties satisfying these modifications of (1), (2), and (3) are called Hausdorff extension properties (a precise definition appears in 2.1). In this paper "Hausdorff extension property" is shortened to "extension property" unless there is a possibility of confusion.

In the remainder of this section we give a brief summary of known results. In $\S 2$ a partial solution to the problem of characterizing Hausdorff extension properties is given (along the lines of conditions (a), (b), and (c)). In addition, certain types of extension properties are classified into broad groups with each group having a largest element, and methods of defining extension properties are developed; this work is similar to the investigations of Tychonoff extension properties carried out in [20]. In the third section the lattice of $\mathscr{P}^{\circ}$-extensions of a fixed space is investigated for certain extension properties $\mathscr{P}$. Results analogous to those of Magill [11] that relate the lattice of compactifications to the Stone-Čech compactification remainder and to the generalizations of Magill's results by Mack, Rayburn, and Woods [12] and Porter [13] are obtained.

All hypothesized topological spaces throughout the paper are assumed to Hausdorff, thus, the word "space" will mean "Hausdorff topological space". A topological property is identified with the class of spaces possessing it, e.g., if $\mathscr{K}$ is the class of compact spaces, that $X$ is compact is also indicated by writing " $X \in \mathscr{K}$ ". The cardinality of a set $S$ is denoted by $|S|$. If $\mathscr{S}$ is a set of sets, then $\bigcup\{S: S \in \mathscr{S}\}$ will be denoted by $\bigcup \mathscr{S}$.

Many of the concepts defined below are also discussed in [15]; the reader is referred to this as a useful source of background material.

DEFINITION 1.1. (a) A space $Y$ is an extension of a space $X$ if $X$ is a dense subspace of $Y$. If $Y$ possesses a topological property $\mathscr{P}$ then $Y$ is a $\mathscr{P}$-extension of $X$. If $Y$ is an extension of $X$ we denote by $1_{X, Y}$ the inclusion map which embeds $X$ as a dense subspace of $Y$.

(b) If $Y$ and $Z$ are extensions of $X$ then a continuous function $f: Y \rightarrow Z$ extends the identity map on $X$ if $f \mid X=1_{X, Z}$. Evidently $f$ extends the identity map on $X$ iff $f$ fixes $X$ pointwise.

We omit the easy proof of the following well-known result.

LEMmA 1.2. Let $Y$ and $Z$ be two extensions of $X$ and let $T$ be an extension of $Y$ (and hence of $X$ ). If $g: Y \rightarrow Z$ and $f: Z \rightarrow T$ are continuous and extend the identity map on $X$ then: 
(a) $\left.f \circ g\right|_{X}=1_{Y, T}$.

(b) $g$ is a homeomorphism from $Y$ onto $g[Y]$ that fixes $X$ pointwise.

Two extensions $Y$ and $Z$ of $X$ are equivalent if there is a homeomorphism from $Y$ onto $Z$ that leaves $X$ pointwise fixed. This is an "equivalence relation" on the class of extensions of $X$. Henceforth we identify equivalent extensions of a space. With this identification the class $\mathscr{E}(X)$ of extensions of $X$ is a set. We next define a partial order on $\mathscr{E}(X)$.

Definition 1.3. (a) Let $Y$ and $Z$ be extensions of $X$. Then $Y$ is projectively larger than $Z$ (denoted $Y \geqq Z$ ) if there is continuous function from $Y$ to $Z$ that fixes $X$ pointwise.

(b) Let $\mathscr{S}$ be a set of extensions of $X$. An extension $Y$ of $X$ is a projective maximum for $\mathscr{S}$ if $Y \in \mathscr{S}$ and $Y \geqq Z$ for all $Z \in \mathscr{S}$.

It is well-known (see, for example, [15]), and follows from 1.2, that $\mathscr{E}(X)$ is partially ordered by $\geqq$. A major theme of this paper is to investigate those topological properties $\mathscr{P}$ for which the set of $\mathscr{P}$-extensions of $X$ has a projective maximum. We give examples of such properties below (see 2.3).

Definition 1.4. A filter (resp. filter base) on the lattice of open sets of $X$ is called an open filter (resp. open filter base) on $X$. A maximal (with respect to set inclusion) element in the set of open filters on $X$ is called an open ultrafilter. The set $\bigcap\left\{\operatorname{cl}_{X} F: F \in \mathscr{F}\right\}$ is called the adherence (in $X$ ) of the open filter base $\mathscr{F}$, and is denoted $\operatorname{ad}_{X}(\mathscr{F})$. An open filter base $\mathscr{F}$ on $X$ is fixed if $\operatorname{ad}_{X}(\mathscr{F}) \neq \varnothing$; otherwise it is free.

Definition 1.5. A space $X$ is $H$-closed if $X$ is closed in each Hausdorff space in which it is embedded. The set of $H$-closed extensions of $X$ will be denoted by $\mathscr{H}(X)$.

The following characterizations of $H$-closed spaces will be useful; see, for example, problems $17 K$ and $17 \mathrm{~L}$ of Willard [19].

THEOREM 1.6. The following are equivalent for a space $X$ :

(a) $X$ is $H$-closed.

(b) Each open ultrafilter on $X$ is fixed.

(c) Each open filter on $X$ is fixed.

(d) If $\mathscr{C}$ is an open cover of $X$ there is a finite subfamily $\mathscr{F}$ of $\mathscr{C}$ such that $\cup \mathscr{F}$ is dense in $X$. 
Recall that a subspace $A$ of a space $X$ is regular closed (resp. regular open) in $X$ if $A=\mathrm{cl}_{X} V$ for some open subset $V$ of $X$ (resp. $A=\operatorname{int}_{X} E$ for some closed subset $E$ of $X$ ). If $\mathscr{P}$ is a topological property such that regular closed subspaces of spaces with $\mathscr{P}$ have $\mathscr{P}$, we say that $\mathscr{P}$ is regular closed hereditary. We omit the easy proof of the following facts.

Proposition 1.7. (a) The property of being H-closed is regular closed hereditary.

(b) Continuous images of H-closed spaces are H-closed.

(c) Regular $H$-closed spaces are compact.

Definition 1.8. (a) Let $X$ be a space and let $X^{*}=X \cup\left\{\mathscr{U}_{6}\right.$ : $\mathscr{U}$ is a free open ultrafilter on $X$ \}. For each open subset $U$ of $X$ let $O_{U}^{\prime}=U \cup\left\{\mathscr{C} \in X^{*} \backslash X: U \in \mathscr{C}\right\} . \quad X^{*}$ with the topology generated by the open base $\left\{O_{U}^{\prime}: U\right.$ open in $\left.X\right\}$ is denoted by $\sigma X$ and called the Fomin extension of $X$ (see $[7,16]$ ).

(b) $X^{*}$ with the topology generated by the open base $\{U: U$ open in $X\} \cup\left\{\{\mathscr{C}\} \cup U: \mathscr{U}_{6} \in X^{*} \backslash X\right.$ and $\left.U \in \mathscr{U}_{6}\right\}$ is denoted by $\kappa X$ and called the Katetov extension of $X$ (see [9]).

We summarize the basic properties of $\kappa X$ in the next theorem.

THEOREM 1.9. (a) $\kappa X$ is a projective maximum for $\mathscr{H}(X)$.

(b) If $Y$ is an extension of $X$ there is a continuous function $f: \kappa X \rightarrow \kappa Y$ that extends the identity map on $X$; explicitly, if $\alpha \in \kappa X \backslash X$ then $f(\alpha)=\bigcap\left\{\mathrm{cl}_{\kappa Y} V: V \in \alpha\right\}$.

(c) If $X \subseteq T$ : $\Subset X$ then $\kappa T=\kappa X$.

(d) If $X \subseteq T \subseteq \kappa X$ and $Y$ is an extension of $X$, then $T \geqq Y$ iff $f[T] \subseteq Y$, where $f: \kappa X \rightarrow \kappa Y$ extends the identity map on $X$.

(e) If $A$ is a regular closed subset of $X$ then $\mathrm{cl}_{\kappa X} A=\kappa A$.

Proof. (a) See [7] and [14].

(b) This follows from (a) and the fact that $\kappa Y$ is an $H$-closed extension of $X$.

(c) See Theorem $\mathrm{E}$ of [5].

(d) Let $f: \kappa X \rightarrow \kappa Y$ extend the identity map to $\kappa X$. If $f[T] \subset Y$, then $f \mid T$ maps $T$ to $Y$ and fixes $X$ pointwise, so $T \geqq Y$. Conversely if $T \geqq Y$ there exists a continuous function $g: T \rightarrow Y$ fixing $X$ pointwise. Let $f: \kappa X \rightarrow \kappa Y$ be the extension of the identity map. Then $f \mid T$ and $g$ both map $T$ into the Hausdorff space $\kappa Y$, and agree on the dense subspace $X$. Thus $f \mid T=g$ and so $f[T] \subset Y$.

(e) This follows from Theorem $\mathrm{F}$ of [5] and Theorem 3.4 of [22]. 


\section{Hausdorff extension properties.}

Definition 2.1. A topological property $\mathscr{P}$ is called an extension property if each $H$-closed space has $\mathscr{P}$ and if, for any space $X$, the set of $\mathscr{P}$-extensions of $X$ has a projective maximum. This projective maximum is denoted by $\kappa_{S} X$.

Proposition 2.2. Let $\mathscr{P}$ be an extension property and let $X$ be $a_{\alpha}^{5}$ space. Then:

(a) $X \subseteq \kappa_{\mathscr{O}} X \leqq \kappa X$.

(b) $\kappa_{\mathscr{X}} X=\bigcap\{T: X \leqq T \leqq \kappa X$ and $T$ has $\mathscr{P}\}$.

Proof. (a) Since $\kappa X$ is a $\mathscr{P}$-extension of $X$ there is a continuous function $g: \kappa_{\mathscr{S}} X \rightarrow \kappa X$ that fixes $X$ pointwise. As $\kappa\left(\kappa_{\mathscr{O}} X\right)$ is an $H$-closed extension of $X$, by 1.9 there is a continuous function $f: \kappa X \rightarrow \kappa\left(\kappa_{\mathscr{S}} X\right)$ that fixes $X$ pointwise. By $1.2(\mathrm{~b}) g$ is a homeomorphism from $\kappa_{,} X$ onto $g\left[\kappa_{,} X\right]$ that fixes $X$ pointwise, so $\kappa_{,} X$ and $g\left[\kappa_{Y} X\right]$ are equivalent extensions of $X$, and thus are identified. As $X \subseteq g\left[\kappa_{\mathscr{O}} X\right] \subseteq \kappa X$ the result follows.

(b) Since $\kappa_{\mathscr{S}} X$ has $\mathscr{P}$ it sufficies to show that if $X \leqq T \leqq \kappa X$ and $T$ has $\mathscr{P}$ then $\kappa_{\mathscr{O}} X \subseteq T$. As $T$ is a $\mathscr{P}$-extension of $X$ there is a continuous function $j: \kappa, X \rightarrow T$ that fixes $X$ pointwise. But $1_{T, \kappa X} \circ j$ and $1_{\kappa_{S} X, \kappa X}$ both map $\kappa_{,} X$ into $\kappa X$ and fix $X$ pointwise. Thus these maps are equal, and so $\kappa_{,} X \subseteq T$.

EXAMPLES 2.3. (a) The property of being $H$-closed is an extension property; $\kappa X$ is the projective maximum of the set of $H$-closed extensions of $X$.

(b) An open filter $\mathscr{F}$ has the closed countable intersection property (C.C.I.P.) if the intersection of the closures of each countable subfamily of $\mathscr{F}$ has a nonempty intersection. A space $X$ is almost realcompact if no free open ultrafilter on $X$ has C.C.I.P. Almost realcompactness is an extension property; an explicit construction of the maximum almost realcompact extension of a space is given in [10].

(c) Let $\mathscr{P}$ be an extension property and let $\mathscr{R}$ be a densehereditary topological property (i.e., if $X$ is dense in $T$ and $T$ has $\mathscr{R}$ then $X$ has $\mathscr{R}$ ). Define the topological property $\mathscr{P} \vee \mathscr{R}$ as follows: $X \in \mathscr{P} \vee \mathscr{R}$ if $X \in \mathscr{P}$ or $X \in \mathscr{R}$. Then $\mathscr{P} \vee \mathscr{R}$ is an extension property. To prove this, if $X$ is a space define the space $X_{\mathscr{O} \vee \Omega}$ as follows: $X_{\mathscr{S} \vee \mathscr{a}}=X$ if $X \in \mathscr{R}$, and $X_{\mathscr{O} \vee a}=\kappa, X$ if $X \notin \mathscr{R}$. Evidently $X_{\mathscr{P} \vee}$ is a $\mathscr{P} \vee \mathscr{R}$-extension of $X$; we will show that it is the projective maximum of the set of $\mathscr{P} \vee \mathscr{R}$-extensions.

If $T$ is a $\mathscr{P} \vee \mathscr{R}$-extension of $X$, then $T \in \mathscr{P}$ or $T \in \mathscr{R}$. If 
$T \notin \mathscr{R}$ then $T \in \mathscr{P}$. As $X_{\mathscr{O} \vee \mathscr{G}}$ is either $\kappa_{\mathscr{G}} X$ or $X$, there is a continuous function $f: X_{\mathscr{S} \vee \mathscr{R}} \rightarrow T$ that fixes $X$ pointwise. Thus $X_{\mathscr{S} \vee \mathscr{R}} \geqq T$. If $T \in \mathscr{R}$ then $X \in \mathscr{R}$ so $1_{X, T}$ maps $X_{\mathscr{O} \vee \mathscr{R}}$ to $T$ and fixes $X$ pointwise.

We will consider the following two special cases of (c) in some detail in 2.16 and 2.17 .

(d) If $\mathscr{P}$ is " $H$-closed" and $\mathscr{R}$ is "Tychonoff", then $X \in \mathscr{P} \vee \mathscr{R}$ iff either $X$ is $H$-closed or $X$ is Tychonoff. We denote $\mathscr{P} \vee \mathscr{R}$ in this case by $\mathscr{P}_{0}$.

(e) If $\mathscr{P}$ is " $H$-closed" and $\mathscr{R}$ is "contains an isolated point" then we denote $\mathscr{P} \vee \mathscr{R}$ by $\mathscr{P}_{1}$.

In the remainder of this section we attempt to develop a systematic theory of extension properties similar to that developed for Tychonoff extension properties in [6] and [20]. Unfortunately properties such as $\mathscr{P}_{0}$ and $\mathscr{P}_{1}$ defined above turn out to be quite "badly behaved"; as a result the theory of Hausdorff extension properties is not as well structured as the corresponding theory of Tychonoff extension properties.

The authors would like to thank J. Vermeer for bringing the existence of $\mathscr{P}_{0}$ and $\mathscr{P}_{1}$, together with their properties as discussed below, to their attention.

We now define several properties that a topological property can have. These properties will be of importance when we study the structure of extension properties and ways of generating new extension properties.

DEFinition 2.4. Let $\mathscr{P}$ be a topological property.

(a) $\mathscr{P}$ has the $\kappa$-inversion property (respectively, weak $\kappa$-inversion property) if, whenever $Y$ is a $\mathscr{P}$-extension of $X$ and $f: \kappa X \rightarrow \kappa Y$ extends the identity map, then $f^{-}[Y]$ has $\mathscr{P}$ (respectively, there exists a $\mathscr{P}$-extension $T$ of $X$ such that $\left.X \subset T \subset f^{\leftarrow}[Y]\right)$.

(b) $\mathscr{P}$ has the $\kappa$-epimorphism property if, whenever $X \subseteq T \subseteq$ $\kappa X, Y$ is an extension of $X, T \geqq Y$, and $T$ has $\mathscr{P}$, then $Y$ has $\mathscr{P}$.

(c) $\mathscr{P}$ has the $\kappa$-intersection property if, whenever $\left(T_{i}\right)_{i \in I}$ is a family of $\mathscr{P}$-extensions of $X$ with $X \subseteq T_{i} \subseteq \kappa X$ for each $i \in I$, then $\bigcap\left\{T_{i}: i \in I\right\}$ has $\mathscr{P}$.

(d) $\mathscr{P}$ is determined by dense subspaces if, whenever $X$ has $\mathscr{P}$ and $X$ is a dense subspace of $T$, then $T$ has $\mathscr{P}$.

THEOREM 2.5. The following are equivalent for a topological property $\mathscr{P}$.

(a) $\mathscr{P}$ is an extension property.

(b) All H-closed spaces have $\mathscr{P}, \mathscr{P}$ has the $\kappa$-intersection property, and $\mathscr{P}$ has the weak $\kappa$-inversion property. 
Proof. $\quad(a) \Rightarrow$ (b). By definition each $H$-closed space has $\mathscr{P}$ if $\mathscr{P}$ is an extension property. Let $\left(T_{i}\right)_{i \in I}$ be a family of $\mathscr{P}$-extensions of $X$ with $X \subseteq T_{i} \leqq \kappa X$. Let $T=\bigcap_{i \in I} T_{i}$; then $X \subseteq T \leqq \kappa X$ so by 1.9 (c) $\kappa T=\kappa X$. Thus by $2.2(\mathrm{a}) T \leqq \kappa_{5} T \subseteq \kappa X$. If $T \neq \kappa_{0} T$ then there exists $i_{0} \in I$ with $T \leqq T_{i_{0}} \cap \kappa_{0} T \varsubsetneqq \kappa_{0} T$. As $T_{i_{0}}$ is a $\mathscr{P}$-extension of $T$, there is a continuous function $f: \kappa, T \rightarrow T_{i_{0}}$ such that $f \mid T=1_{T}$. This is obviously impossible, so $T=\kappa, T$ and $T$ has $\mathscr{P}$. Hence $\mathscr{P}$ has the $\kappa$-intersection property. Finally let $Y$ be a $\mathscr{P}$-extension of $X$ and let $f: \kappa X \rightarrow \kappa Y$ extend the identity map to $\kappa X$. There is a continuous function $g: \kappa, \rightarrow Y$ such that $g \mid X=1_{X}$. Since $X \subseteq$ $\kappa_{\Rightarrow} X \subseteq \kappa X$, it follows that $f \mid \kappa_{,} X=g$. Thus $X \subseteq \kappa_{,} X \subseteq f^{\leftarrow}[Y]$ and $\kappa_{,} X$ has $\mathscr{P}$. Hence $\mathscr{P}$ has the weak $\kappa$-inversion property.

(b) $\Rightarrow$ (a). For each space $X$ let $\mathscr{C}(X)=\{T: X \leqq T \subseteq \kappa X$ and $T$ has $\mathscr{P}\}$. Since each $H$-closed space has $\mathscr{P}, \mathscr{C}(X) \neq \varnothing$. Let $Z=\bigcap\{T: T \in \mathscr{C}(X)\}$. As $\mathscr{P}$ has the $\kappa$-intersection property, $Z$ has $\mathscr{P}$. We now claim that $Z$ is the projective maximum of the set of $\mathscr{P}$-extension of $X$. Let $Y$ be a.P्P-extension of $X$, and let $f: \kappa X \rightarrow \kappa Y$ extend the identity map on $X$. As $\mathscr{P}$ has the weak $\kappa$-inversion property there is a space $W$ with $\mathscr{P}$ such that $X \subseteq W \subseteq f^{\leftarrow}[Y]$. As $W \in \mathscr{C}(X), Z \subseteq W$. Thus $f \mid Z$ maps $Z$ continuously into $Y$ and extends the identity map. Thus $Z=\kappa, X$ and $\mathscr{P}$ is an extension property.

There is a "Tychonoff analogue" of the $\kappa$-inversion property as follows: if $\mathscr{P}$ is a Tychonoff extension property, $Y$ has $\mathscr{P}, f: X \rightarrow Y$ is continuous, and $f^{\beta}: \beta X \rightarrow \beta Y$ is the extension of $f$ to the StoneCech compactification, then $\left(f^{\beta}\right)^{-}[Y]$ has $\mathscr{P}$. This, in fact, is true of all Tychonoff extension properties (see [6]) and is used extensively in developing the theory of Tychonoff extension properties (see [20]). Unfortunately not all Hausdorff extension properties have the $\kappa$-inversion property (see 2.16 below). However, regular-closed hereditary Hausdorff extension properties with the $\kappa$-inversion property (such as almost realcompactness) behave much like Tychonoff extension properties. For this reason we pay considerable attention to such Hausdorff extension properties in what follows.

Proposition 2.6. The following are equivalent for an extension property $\mathscr{P}$ :

(a) $\mathscr{P}$ has the $\kappa$-inversion property.

(b) If $X \subseteq T \subseteq \kappa X, Y$ is an extension of $X$, and $T \geqq Y$, then $\kappa_{\mathscr{S}} T \geqq \kappa_{\mathscr{S}} Y$.

(c) If eX is a $\mathscr{P}$-extension of $X$ then $\mathrm{cl}_{\kappa X \times e X} \Delta_{X}$ has $\mathscr{P}$ (where $\Delta_{X}=\{(x, x): x \in X\}$, viewed as a subspace of $\left.\kappa X \times e X\right)$. 
Proof. (a) $\Rightarrow(\mathrm{b})$. Let $f: \kappa X \rightarrow \kappa Y$ extend the identity map Since $T \geqq Y, f[T] \leqq Y$. By $2.2(\mathrm{a}) \kappa_{\mathscr{S}} Y \leqq \kappa Y$, so by hypothesis $f^{\leftarrow}\left[\kappa_{\mathscr{O}} Y\right]$ has $\mathscr{P}$, and contains $T$. Thus by $2.2(\mathrm{~b}) \kappa_{\mathscr{S}} T \leqq f^{\leftarrow}\left[\kappa_{\mathscr{S}} Y\right]$, so $f \mid \kappa_{\mathscr{O}} T$ maps $\kappa_{\mathscr{O}} T$ into $\kappa_{\mathscr{S}} Y$ and fixes $X$ pointwise. Thus

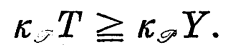

(b) $\Rightarrow$ (a). Let $Y$ be a $\mathscr{P}$-extension of $X$ and let $f: \kappa X \rightarrow \kappa Y$ extend the identity map on $X$. Then $f^{\leftarrow}[Y] \geqq Y$, so by hypothesis $\kappa_{-} f[Y] \geqq \kappa_{,} Y=Y$. Thus there exists a continuous function $g: \kappa_{S} f^{\leftarrow}[Y] \rightarrow Y$ such that $g \mid X=1_{X}$. By $2.2(\mathrm{a})$ and $1.9(\mathrm{c}) f^{\leftarrow}[Y] \subseteq$ $\kappa_{\mathscr{O}} f \leftarrow[Y] \subseteq \kappa X$, so $g$ and $f \mid \kappa_{\mathscr{S}} f^{\leftarrow}[Y]$ both map $\kappa_{\mathscr{P}} f^{\leftarrow}[Y]$ into $\kappa Y$ and agree on $X$. Hence $g=f \mid \kappa_{\mathscr{S}} f^{\leftarrow}[Y]$ so $f\left[\kappa_{\mathscr{O}} f^{\leftarrow}[Y]\right] \subseteq Y$. This implies that $\kappa_{\mathscr{P}} f^{\leftarrow}[Y]=f^{\leftarrow}[Y]$, so $Y$ has $\mathscr{P}$. Thus $\mathscr{P}$ has the $\kappa$-inversion property.

(c) $\Leftrightarrow$ (a). Let $f: \kappa X \rightarrow \kappa(e X)$ extend the identity map on $X$. It is straightforward to show that $\operatorname{cl}_{\wedge X \times e X} \Delta_{X}=\left\{(x, f(x)): x \in f^{\leftarrow}[e X]\right\}$ and that this latter set is homeomorphic to $f^{-}[e X]$. The equivalence follows.

Definition 2.7. Let $\mathscr{P}$ be a topological property. A space $X$ is said to be $\mathscr{P}$-pseudo- $H$-closed if each extension of $X$ with $\mathscr{P}$ is

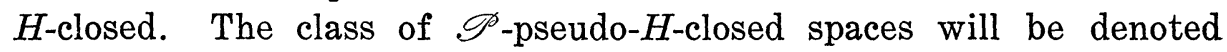
by $\mathscr{P}^{\prime}$.

The definition of $\mathscr{P}$-pseudo- $H$-closed spaces is similar to the definition of $\mathscr{P}$-pseudocompact spaces (for Tychonoff extension properties $\mathscr{P}$ ) introduced in [20]; hence the choice of terminology. Each part of the following lemma has a one-line proof.

Lemma 2.8. Let $\mathscr{P}$ and $\mathscr{Q}$ be topological properties. Then

(a) $\mathscr{P}^{\prime}$ is determined by dense subspaces.

(b) If all $H$-closed spaces have $\mathscr{P}$, then $X$ is $H$-closed iff $X$ has both $\mathscr{P}$ and $\mathscr{P}^{\prime}$.

(c) If $\mathscr{P} \subseteq \mathbb{Q}^{2}$ then $\mathbb{Q}^{\prime} \subseteq \mathscr{P}^{\prime}$.

(d) If $\mathscr{P}$ is an extension property then $X$ has $\mathscr{P}^{\prime}$ iff $\kappa_{\mathscr{O}} X$ is H-closed.

LEMma 2.9. If $\mathscr{P}$ is a topological property with the $\kappa$-inversion property, then $\mathscr{P}^{\prime}$ has the k-epimorphism property.

Proof. Let $T$ be a $\mathscr{P}^{\prime}$-extension of $X$ with $X \subseteq T \leqq \kappa X$, let $Y$ be an extension of $X$, and let $T \geqq Y$. We must show $Y$ has $\mathscr{P}^{\prime}$. Let $Z$ be an extension of $Y$ with $\mathscr{P}$; we must show that $Z$ is $H$ - 
closed. Let $f: \kappa X \rightarrow \kappa Z$ extend the identity map on $X$, let $g: \kappa Y \rightarrow$ $\kappa Z$ extend the identity map on $Y$, and let $h: \kappa X \rightarrow \kappa Y$ extend the identity map on $X$. Then $g \circ h$ maps $\kappa X$ to $\kappa Z$ and agrees with $f$ on $X$, so $f=g \circ h$. As $\mathscr{P}$ has the $\kappa$-inversion property, $f^{\leftarrow}[Z]$ has $\mathscr{P}$, i.e., $h^{\leftarrow}\left[g^{-}[Z]\right]$ has $\mathscr{P}$. Now $Y \subset g^{-}[Z]$ and $h[T] \subset Y$ since $T \geqq Y$; thus $T \subset f^{\leftarrow}[Z]$. As $T$ has $\mathscr{P}^{\prime}, f^{\leftarrow}[Z]$ is $H$-closed. Thus by $1.7(\mathrm{~b})$ $Z$ is $H$-closed and so $Y$ has $\mathscr{P}^{\prime}$.

We now describe a general method of constructing extension properties. The procedure consists of starting with a topological property $\mathscr{R}$ that is determined by dense subspaces and satisfies the $\kappa$-epimorphism property, and defining an extension property $\hat{\mathscr{R}}$ in terms of $\mathscr{R}$. By $2.8(a)$ and 2.9 if $\mathscr{P}$ is an extension property with the $\kappa$-inversion property, then $\mathscr{P}^{\prime}$ is determined by dense subspaces and satisfies the $\kappa$-epimorphism property, and so $\widehat{\mathscr{P}}^{\prime}$ is an extension property whose relationship to the original extension property $\mathscr{P}$ becomes a subject of interest.

Definition 2.10. Let $\mathscr{R}$ be a topological property. The topological property $\hat{\mathscr{R}}$ is defined as follows: $X$ has $\hat{\mathscr{R}}$ if each regular closed subset of $X$ with $\mathscr{R}$ is $H$-closed.

THEOREM 2.11. Let $\mathscr{R}$ be any topological property. Then:

(a) All H-closed spaces have $\hat{\mathscr{R}}$ and $\hat{\mathscr{R}}$ is regular closed hereditary.

(b) Let $T$ and $\left\{T_{i}: i \in I\right\}$ be a collection of extensions of a space $X$ such that $X \subset T_{i} \subset T$ for each $i$ and $X=\bigcap\left\{T_{i}: i \in I\right\}$. If $\mathscr{R}$ is determined by dense subspaces and each $T_{i}$ has $\hat{\mathscr{R}}$ then $X$ has $\hat{\mathscr{R}}$. In particular $\hat{\mathscr{R}}$ has the $\kappa$-intersection property.

(c) If $\mathscr{R}$ has the $\kappa$-epimorphism property and is determined by dense subspaces, then $\hat{\mathscr{R}}$ has the $\kappa$-inversion property and hence is an extension property.

Proof. (a) That all $H$-closed spaces have $\hat{\mathscr{R}}$ follows from 1.7(a). If $A$ is a regular closed subset of $X$ and $B$ is a regular closed subset of $A$, then $B$ is a regular closed subset of $X$; it immediately follows that $\hat{\mathscr{R}}$ is regular closed hereditary.

(b) Let $A$ be a regular closed subspace of $X$ with $\mathscr{R}$. For each $i \in I, \operatorname{cl}_{T_{i}} A$ is a regular closed subset of $T_{i}$, and has $\mathscr{R}$ as $\mathscr{R}$ is determined by dense subspaces. Since $T_{i}$ has $\hat{\mathscr{R}}, \operatorname{cl}_{T_{i}} A$ is $H$ closed. Thus $\mathrm{cl}_{T_{i}} A=\mathrm{cl}_{{ } T}\left(\mathrm{cl}_{T_{i}} A\right)=\mathrm{cl}_{\kappa T} A$ for each $i \in I$. Thus $A=$ $\bigcap\left\{\mathrm{cl}_{T_{i}} A: i \in I\right\}=\mathrm{cl}_{\kappa T} \dot{A}$. Thus $A$ is $\dot{H}$-closed and $X$ has $\hat{\mathscr{R}}$.

(c) By (a) and (b) all $H$-closed spaces have $\hat{\mathscr{R}}$ and $\hat{\mathscr{R}}$ has the 
$\kappa$-intersection property. Thus once it is shown that $\hat{\mathscr{R}}$ has the $\kappa$-inversion property, it follows from 2.5 that $\hat{\mathscr{R}}$ is an extension property.

Let $r X$ be an $\hat{\mathscr{R}}$-extension of $X$ and let $f: \kappa X \rightarrow \kappa(r X)$ extend the identity map. We must show that $f \leftarrow[r X]$ has $\hat{\mathscr{R}}$. Let $A$ be a regular closed subspace of $f^{\leftarrow}[r X]$ with $\mathscr{R}$. Then $A \cap X$ is a regular closed subspace of $X$; so, by $1.9(\mathrm{e}), \mathrm{cl}_{\kappa X}(A \cap X)=\kappa(A \cap X)$. Thus $A \cap X \subset A \subseteq \kappa(A \cap X)$. As $f[A]$ is an extension of $A \cap X$ and $f \mid A$ extends the identity function on $A \cap X$, we have $A \geqq f[A]$ in the partial ordering of extensions of $A \cap X$. Since $\mathscr{R}$ has the $\kappa$-epimorphism property, it follows that $f[A]$ has $\mathscr{R}$.

There is an open subset $V$ of $r X$ such that $V \cap X=\operatorname{int}_{X}(A \cap X)$. As $A \cap X$ is dense in $f[A]$, it follows that

$$
\operatorname{cl}_{r X} V=\operatorname{cl}_{r X}(V \cap X)=\operatorname{cl}_{r X}(A \cap X)=\operatorname{cl}_{r X} f[A] .
$$

Since $f[A]$ has $\mathscr{R}$ and $\mathscr{R}$ is determined by dense subspaces, $\operatorname{cl}_{r X} f[A]$ is a regular closed subset of $r X$ with $\mathscr{R}$. As $r X$ has $\hat{\mathscr{R}} \operatorname{cl}_{r X} f[A]$ is $H$-closed. If $A$ were not $H$-closed then $\operatorname{cl}_{X} A-A \neq \varnothing$, and so $\operatorname{cl}_{X} A-f \leftarrow[r X] \neq \varnothing$. Thus $f\left[\operatorname{cl}_{X} A\right]-r X \neq \varnothing$, and so $\operatorname{cl}_{X} f[A]$ is a proper dense subspace of $f\left[\mathrm{cl}_{X} A\right]$. This contradicts the fact that $\operatorname{cl}_{X} f[A]$ is $H$-closed. Hence $A$ is $H$-closed, $\hat{\mathscr{R}}$ has the $\kappa$-inversion property, and hence $\hat{\mathscr{R}}$ is an extension property.

The most important special case of 2.11 arises when the $\mathscr{R}$ of 2.11 is $\mathscr{P}^{\prime}$ for a suitably chosen property $\mathscr{P}$. We will show that under certain conditions $\widehat{\mathscr{P}}$ ' is a "well-behaved" extension property that is the "largest" extension property belonging to a certain class. Note that a space $X$ has $\widehat{\mathscr{P}^{\prime}}$ iff whenever $A$ is a regular closed subset of $X$ that is not $H$-closed, then $A$ has $\mathscr{P}$-extensions that are not $H$-closed.

LemMa 2.12. Let $\mathscr{P}$ be a topological property. Then:

(a) If $\mathscr{P}$ has the $\kappa$-inversion property then $\widehat{\mathscr{P}}^{\prime}$ is a regular closed hereditary extension property with the $\kappa$-inversion property.

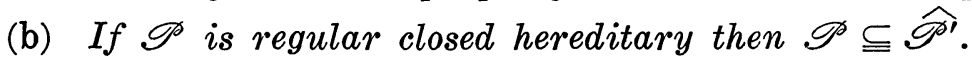

(c) If $\mathscr{P}$ is regular closed hereditary and has the $\kappa$-inversion property then $\mathscr{\mathscr { P }}^{\prime}=\left(\widehat{\mathscr{P}^{\prime}}\right)^{\prime}$.

(d) If $\mathscr{P}$ has the $\kappa$-epimorphism property and is determined by dense subspaces then $\mathscr{\mathscr { P }} \cong(\widehat{\mathscr{P}})^{\prime}$ and $\widehat{\mathscr{P}}=\widehat{(\widehat{\mathscr{P}})^{\prime}}$.

Proof. (a) This follows from 2.8(a), 2.9, 2.11(a) and 2.11(c). 
(b) Suppose $X$ has $\mathscr{P}$ and $A$ is a regular closed subset of $X$ with $\mathscr{P}^{\prime}$. Then each $\mathscr{P}$-extension of $A$ is $H$-closed. By hypothesis $A$ itself is such an extension, so $A$ is $H$-closed. Thus $X$ has $\widehat{\mathscr{P}^{\prime}}$.

(c) By 2.8 (c) and (b) above, $\left(\widehat{\mathscr{P}}^{\prime}\right)^{\prime} \subset \mathscr{P}^{\prime}$. By (i) above $\mathscr{P}$ is an extension property. If $X$ has $\mathscr{P}^{\prime}$ then by $2.8\left(\right.$ a) $\kappa_{\hat{\prime}} X$ has $\mathscr{P}^{\prime}$. But $\kappa_{\hat{\mathcal{P}}} X$ is a regular closed subset of itself, so by definition of $\widehat{\mathscr{P}^{\prime}}$, $\kappa_{\hat{\mathscr{F}}} X$ is $H$-closed. Thus by $2.8(\mathrm{~d}) X$ has $\left(\widehat{\mathscr{P}^{\prime}}\right)^{\prime}$ and $\mathscr{\mathscr { P }}^{\prime} \cong\left(\widehat{\mathscr{P}^{\prime}}\right)^{\prime}$.

(d) Suppose $X$ has $\mathscr{P}$. By $2.11($ c) $\hat{\mathscr{P}}$ is an extension property and $\kappa_{\hat{y}} X$ is an extension of $X$ with $\hat{\mathscr{P}}$. Since $\mathscr{P}$ is determined by dense subspaces, $\kappa_{\hat{s}} X$ also has $\mathscr{P}$. As $\kappa_{\hat{g}} X$ is a regular closed subset of itself, it follows that $\kappa_{\hat{\mathscr{S}}} X$ is $H$-closed and so $X$ has $(\hat{\mathscr{P}})^{\prime}$ by $2.8(\mathrm{~d})$. Thus $\mathscr{P} \cong(\widehat{\mathscr{P}})^{\prime}$. It is easily seen that if $\mathscr{R}$ and $\mathscr{S}$ are two topological properties with $\mathscr{R} \subseteq \mathscr{S}$, then $\hat{\mathscr{S}} \subseteq \widehat{\mathscr{R}}$; thus $\widehat{(\widehat{\mathscr{P}})^{\prime}} \subseteq \widehat{\mathscr{P}}$. Conversely, by $2.11(\mathrm{a}) \hat{\mathscr{P}}$ is regular closed hereditary so by (b) $\mathscr{P}^{\prime} \subseteq$ $\widehat{(\hat{\mathscr{P}})^{\prime}}$. Thus $\hat{\mathscr{P}}=\widehat{(\widehat{\mathscr{P}})^{\prime}}$.

Definition 2.13. (a) Two topological properties $\mathscr{P}$ and $\mathbb{Q}$ are co-pseudo-H-closed if $\mathscr{P}^{\prime}=\mathscr{Q}^{\prime}$. The class $\left\{\mathscr{Q}^{\prime}: \mathscr{Q}^{\prime}=\mathscr{P}^{\prime}\right\}$ is called the co-pseudo-H-closed class of the property $\mathscr{P}$.

(b) An extension property is called strong extension property if it is regular closed hereditary and has $\kappa$-inversion property.

Being co-pseudo- $H$-closed is an equivalence relation on the class of topological properties. In the next theorem, which is the main result of $\S 2$, we show that if $\mathscr{C}(\mathscr{P})$ is the class of all strong extension properties co-pseudo- $H$-closed with the strong extension property $\mathscr{P}$, then $\mathscr{C}(\mathscr{P})$ has a largest member, namely $\widehat{\mathscr{P}^{\prime}}$. This parallels similar results obtained in [20] for Tychonoff extension properties. As Example 2.17 shows, this attractive result fails for arbitrary extension properties.

THEOREM 2.14. (a) If $\mathscr{P}$ is a strong extension property, then $\widehat{\mathscr{P}^{\prime}}$ is a strong extension property co-pseudo-H-closed with $\mathscr{P}$ and containing all other strong extension properties co-pseudo-H-closed with $\mathscr{P}$.

(b) If $\mathscr{R}$ is a topological property determined by dense subspaces and possessing the $\kappa$-epimorphism property, then $\hat{\mathscr{R}}$ is a strong extension property and is the largest member of $\mathscr{C}(\hat{\mathscr{R}})$.

Proof. Part (a) follows from 2.12(a), (b), and (c), and part (b) follows from $2.11(\mathrm{c})$ and $2.12(\mathrm{~d})$. 
In the remainder of this section we consider a variety of examples illustrating the applications and limitations of the above theory.

EXAMPLE 2.15. Almost realcompactness is an extension property (see 2.3(b)) that is regular closed hereditary (see [3]). Almost realcompactness has the $\kappa$-inversion property (the verification of this, using $1.9(\mathrm{~b})$, is tedious but routine). Thus almost realcompactness is a strong extension property; denote it by $\mathscr{P}$. Then by 2.14 (a) $\widehat{\mathscr{P}}^{\prime}$ is a strong extension property co-pseudo- $H$-closed with $\mathscr{P}$ and containing $\mathscr{P}$. If $X$ is a (Tychonoff) non-almost realcompact $P$-space, then $X \in \widehat{\mathscr{P}^{\prime}}-\mathscr{P}$ (recall that a space $X$ is a $P$-space if its $G_{\tilde{\delta}}$-sets are open; see. Problems $4 \mathrm{~K}$ and $4 \mathrm{~L}$ of [4] for details). To justify this, note that a Tychonoff space $X$ belongs to $\mathscr{P}^{\prime}$ iff it is pseudocompact (see 3.19). But pseudocompact $P$-spaces are compact (see $4 \mathrm{~K}$ of [4]); thus $\widehat{\mathscr{P}}^{\prime}$ contains all (Tychonoff) $P$-spaces. An example of a Tychonoff $P$-space that is not almost realcompact is given in 9L of [4].

By considering the examples introduced in $2.3(\mathrm{~d})$ and (e) we now show that the hypotheses made on our extension property $\mathscr{P}$ in 2.12 and 2.14 are necessary to the conclusions.

EXAMPle 2.16. Let $\mathscr{R}$ be as in $2.3(\mathrm{c})$ and let $\mathscr{H}$ be the property of being $H$-closed. A routine calculation shows the following: $X \in(\mathscr{H} \vee \mathscr{R})^{\prime}$ iff either $X \notin \mathscr{R}$ or $X$ is $H$-closed and $X \in \overline{(\mathscr{H} \vee \mathscr{R})^{\prime}}$ iff each regular closed subset without $\mathscr{R}$ is $H$-closed.

We now show that the property $\mathscr{P}_{0}$ of $2.3(\mathrm{~d})$ fails to have the $\kappa$-inversion property. Let $N$ be the countable discrete space; then $\kappa N$ and $\beta N$ have the same underlying set of points ( $N$ together with the free ultrafilters on $N$ ) but different topologies. Let $j: \kappa N \rightarrow \beta N$ denote the identity map on the underlying sets. Then $j$ is continuous and extends the identity map on $N$. If $p \in \beta \boldsymbol{N} \backslash \boldsymbol{N}$ then $\beta \boldsymbol{N} \backslash\{p\} \in$ $\mathscr{P}_{0}$, but $j-[\beta N \backslash\{p\}]=\kappa N \backslash\{p\} \notin \mathscr{P}_{0}$, so $\mathscr{P}_{0}$ does not have the $\kappa$-inversion property. This example also shows that $\mathscr{P}_{0}^{\prime}$ does not have the $\kappa$-epimorphism property. However, $\mathscr{P}_{0}^{\prime}$ is easily seen to be regular closed hereditary.

Since $\mathscr{P}_{0}^{\prime}$ does not have the $\kappa$-epimorphism property, 2.11 (c) no longer guarantees that $\widehat{\mathscr{P}_{0}^{\prime}}$ is an extension property, and in fact it is not. To see this, note that for each $p \in \kappa N \backslash N, \kappa N \backslash\{p\} \in \widehat{\mathscr{P}_{0}^{\prime}}$. Hence by $2.2(\mathrm{~b})$ the maximum $\widehat{\mathscr{P}_{0}^{\prime}}$-extension of $N$ (if it exists) must be $N$; however, $N \notin \widehat{\mathscr{P}_{0}^{\prime}}$. Thus $\mathscr{\mathscr { P }}_{0}^{\prime}$ is not an extension property.

EXAMPLE 2.17. It is easily verified that the property $\mathscr{P}_{1}$ of $2.3(\mathrm{e})$ 
is not regular closed hereditary but has the $\kappa$-inversion property. By 2.12 (a) $\widehat{\mathscr{P}_{1}^{\prime}}$ is a regular closed hereditary extension property with the $\kappa$-inversion property. Evidently $X \in \widehat{\mathscr{P}_{1}^{\prime}}$ iff each regular closed subset of $X$ without isolated points is $H$-closed; equivalently, if $X=I(X) \cup H \cup F$ where $I(X)$ is the set of isolated points, $H$ is an $H$-closed subset of $X$, and $F$ is a closed nowhere dense subspace of $X$. In contrast to $2.12(\mathrm{~b}), \mathscr{P}_{1} \nsubseteq \widehat{\mathscr{P}_{1}^{\prime}}$; for example $(0,1) \cup\{2\}$, viewed as a subspace of $\boldsymbol{R}$, belongs to $\widehat{\mathscr{P}}_{1}^{\prime} \backslash \mathscr{P}_{1}$. It is also easy to check that $\widehat{\mathscr{P}_{1}^{\prime}} \varsubsetneqq\left(\widehat{\mathscr{P}_{1}^{\prime}}\right)^{\prime}$.

EXAMPLE 2.18. One might be tempted to conjecture that if $\mathscr{R}$ is a property satisfying the hypotheses of $2.11(\mathrm{c})$, then $(\hat{\mathscr{R}})^{\prime}=$ $\mathscr{R}$. This is untrue; as an example, let $\mathscr{R}$ be the property "is an extension of $N$ or is $H$-closed". Then $\mathscr{R}$ has the required properties, but if $X$ is the free union of the closed unit interval and the space $N \cup\{\alpha\}$, where $\alpha \in \kappa N \backslash N$ then $X \in(\mathscr{\mathscr { R }})^{\prime} \backslash \mathscr{R}$.

2.19. A description of $\kappa_{\mathscr{S}} X$. Sometimes an explicit description of $\kappa_{,} X$ is difficult to obtain, but in special circumstances we can obtain such a description. Let $\mathscr{R}$ be a topological property satisfying the hypotheses of 2.11 (c). Then $\hat{\mathscr{R}}$ is an extension property. For each space $X$ define $\hat{\mathscr{R}} X$ to be $\bigcup\left\{X \cup \operatorname{cl}_{k X} V: V\right.$ is open in $X$ and $\operatorname{cl}_{X} V$ has $\mathscr{R}\}$. A routine verification shows that $X \subset \hat{\mathscr{R}} X \subset \kappa_{\hat{\mathscr{R}}} X$. It follows from $2.2(\mathrm{~b})$ that if $\hat{\mathscr{R}} X$ has $\hat{\mathscr{R}}$, then $\kappa_{\hat{\mathscr{R}}} X=\hat{\mathscr{R}} X$. The reader can easily verify that this occurs if $\mathscr{R}$ is inherited by dense open subspaces. For example, if $m$ is an infinite cardinal and if $\mathscr{R}$ is either "cellularity no greater than $m$ " or "density character no greater than $m$ ", then $\mathscr{R}$ is such a property (see [8] or [17] for a discussion of these and other cardinal invariants).

There are topological properties $\mathscr{R}$ satisfying $2.11(\mathrm{c})$, and not inherited by dense open subspaces, for which $\kappa_{\hat{\mathscr{R}}} X=\hat{\mathscr{R} X}$. We consider two of these.

Let $m$ be an infinite cardinal. A space $X$ is $m$-H-closed if each open cover of $X$ of cardinality no greater than $m$ has a finite subfamily whose union is dense in $X$. A space $X$ is $m$-weakly Lindelof if each open cover of $X$ has a subfamily cardinality no greater than $m$ whose union is dense in $X$. If $m=\aleph_{0}, m$-H-closedness is also called feeble compactness; among Tychonoff spaces $\aleph_{0}-H$-closedness is equivalent to pseudocompactness (see 9.13 of [4]). $\aleph_{0}$-weaklyLindelof spaces are simply called weakly Lindelof spaces; these have 
been studied by Ulmer [18] and Woods [21], among others.

It is easy to show that if $\mathscr{R}$ is either $m$-H-closedness or the $m$-Lindelof property, then $\mathscr{R}$ is determined by dense subspaces, has the $\kappa$-epimorphism property, but is not necessarily preserved by dense open subspaces. Nonetheless, a somewhat lengthy but straightforward calculation shows that $\kappa_{\hat{\mathscr{R}}} X=\hat{\mathscr{R}} X$.

3. Lattices of $\mathscr{P}$-extensions. In this section we develop the machinery to prove that the lattice structure of a certain subset of the tight $\mathscr{P}$-extensions of a Hausdorff space (defined in 3.7) is completely determined by the topological structure of a certain space. This result parallels Magill's result [11], the corresponding result for the lattice of Hausdorff compactifications of a locally compact Hausdorff space, and the generalizations of Magill's result in [12] and is a generalization of Theorem 2.3 in [13], the corresponding result for the upper semilattice of $H$-closed extensions of a space. First, some basic facts about absolutes need to be recalled.

Absolutes 3.1. A function $f: X \rightarrow Y$ is $\theta$-continuous if for each $x \in X$ and open neighborhood $U$ of $f(x)$, there is an open neighborhood $V$ of $x$ such that $f\left(\operatorname{cl}_{X} V\right) \subseteq \operatorname{cl}_{Y} U ; f$ is irreducible if $f$ is onto and no proper closed subset of $X$ is mapped onto $Y$; and $f$ is perfect if $f$ is closed and for each $y \in Y, f^{-}(y)$ is compact. A space is extremally disconnected if its open sets have open closure (see [4]).

For a space $X$, let $\theta X=\{\mathscr{U}: \mathscr{C}$ is open ultrafilter on $X\}$. For an open subset $U$ of $X$, let $O_{U}=\{\mathscr{U} \in \theta X: U \in \mathscr{C}\}$. Now, $\theta X$ with the topology generated by the open basis $\left\{O_{U}: U\right.$ open in $\left.X\right\}$ is a compact Hausdorff, extremally disconnected space and is sometimes denoted by $G X$ and called the Gleason space of $X$ (see [1]).

The subspace $E X=\{\mathscr{C} \in \theta X: \mathscr{C}$ is fixed $\}$ of $\theta X$ is extremally disconnected and Tychonoff and is called the absolute of $X$. Define $\pi: E X \rightarrow X$ by $\pi(\mathscr{C})=\operatorname{ad}_{X} \mathscr{C}$; the surjection $\pi$ is $\theta$-continuous, perfect and irreducible. The absolute $E X$ of $X$ is unique in this sense: if $Y$ is an extremally disconnected, Tychonoff space and there is a $\theta$-continuous, perfect, irreducible surjection $f: Y \rightarrow X$, then there is a homeomorphism $h: E X \rightarrow Y$ such that $f \circ h=\pi$ (see [7]); in such cases, we identify $E X$ and $Y$ and write $E X=Y$. In particular, for a space $X, E(E X)=E X$ by Corollary 2 in [7] and $\theta X=\theta(\theta X)=$ $\theta(E X)=E(\theta X)$ by Theorem 11 in [7]. If two spaces $X$ and $Y$ have homeomorphic absolutes, we say $X$ and $Y$ are co-absolute.

Proposition 3.2. Let $U$ be an open subset of a space $X$.

(a) [7] $X$ is $H$-closed iff $E X$ is compact.

(b) [7] $\pi\left(O_{U} \cap E X\right)=\mathrm{cl}_{X} U$. 
(c) [7] Let $x \in X$. $\pi^{\leftarrow}(x) \subseteq O_{U}$ iff $x \in \operatorname{int}_{X} \operatorname{cl}_{X} U$.

(d) $\left(O_{U} \cap E X, \pi_{U}\right)$ is the absolute of $\operatorname{cl}_{X} U$ where $\pi_{U}$ is $\pi$ restricted to $O_{U} \cap E X$.

Proof of (d). Clearly, $O_{U} \cap E X$ is extremally disconnected and Tychonoff. So, it suffices to show $\pi_{U}: O_{U} \cap E X \rightarrow \mathrm{cl}_{X} U$ is a $\theta$-continuous, perfect, irreducible surjection. It is straightforward to show $\pi_{U}$ is perfect and irreducible; by (b) $\pi_{U}$ is a surjection. To show $\pi_{U}$ is $\theta$-continuous, let $\mathscr{\mathscr { C }} \in O_{U} \cap E X$ and $V$ be an open neighborhood of $\pi_{U}(\mathscr{Q})$ in $\operatorname{cl}_{X} U$. So, there is an open set $W$ in $X$ such that $V=W \cap \mathrm{cl}_{X} V$. Since $\pi_{U}(\mathscr{U}) \in W$, then $W \in \mathscr{U}$. There is an open set $O_{T}$ containing $\mathscr{U}$ such that $\pi\left(O_{T} \cap E X\right) \subseteq \mathrm{cl}_{X} W$. Since $T \in \mathscr{Q}$, we can assume that $T \subseteq U \cap W$. Thus, $O_{r} \subseteq O_{U}$. Let $\mathscr{V} \in$ $O_{T} \cap E X$. Then $T \in \mathscr{V}$ which implies that $\pi(\mathscr{Y}) \in \operatorname{cl}_{X} T \subseteq \operatorname{cl}_{X}(U \cap$ $W)=\operatorname{cl}_{X}\left(\operatorname{cl}_{X} U \cap W\right)=\operatorname{cl}_{X} V$. So, $\pi\left(O_{T} \cap E X\right) \subseteq \operatorname{cl}_{X} V$ and $\pi_{U}$ is $\theta$-continuous.

One of the liabilities of $\theta$-continuity is that the restriction of a $\theta$-continuous function (with the corresponding restriction of the range) may not be $\theta$-continuous (see the remark after 3.4 in [2]). However, as proven in $3.2(\mathrm{~d})$, some restrictions are $\theta$-continuous; such is the case in the following result.

Lemma 3.3. Let $f: X \rightarrow Y$ be a surjection, $S \subseteq X, D \subseteq Y$, $f(S) \cong D$, and $\left.f\right|_{s}: S \rightarrow D$.

(a) If $f$ is $\theta$-continuous and both $S$ and $D$ are dense, then $\left.f\right|_{S}$ is $\theta$-continuous.

(b) If $f$ is perfect and $S=f^{\leftarrow}(D)$, then $\left.f\right|_{S}$ is perfect.

(c) If $f$ is closed and irreducible, $S=f^{\leftarrow}(D)$, and $D$ is dense, then $\left.f\right|_{s}$ is closed and irreducible.

Proof. Straightforward.

Proposition 3.4. Let $h X$ be a $H$-closed extension of a space $X$. Define $\pi_{h}: \theta X \rightarrow h X$ by $\left\{\pi_{h}(\mathscr{Q})\right\}=\operatorname{ad}_{h X} \mathscr{\mathscr { C }}$. Let $X \cong Y \subseteq h X$ and $Z=\pi_{h}^{\leftarrow}(Y)$. Then $\left.\pi_{h}\right|_{Z}: Z \rightarrow Y$ is a $\theta$-continuous, perfect, irreducible surjection; in particular, $Z$ is the absolute of $Y$.

Proof. The proof uses 3.3 and is similar to the format of the proof of Theorem 10 in [7].

Let $Y$ be an $H$-closed extension of $X$ and $p \in Y$. Let $O(p)$ denote the filter base of open neighborhood traces of $p$ on $X$. By 1.9 , let $f: \kappa X \rightarrow Y$ be the unique continuous function that extends 
the identity function on $X$; it is easy to verify for $\mathscr{Q} \in \kappa X \backslash X$, that $\{f(\mathscr{C})\}=\operatorname{ad}_{Y} \mathscr{W}$ and $\mathscr{C} \supseteqq O(f(\mathscr{C}))$. Since $\left.f\right|_{X}=1_{X}$, then using the notation of 3.4, $\left.f \circ \pi_{\kappa}\right|_{E X}=\left.\pi_{Y}\right|_{E X}$. If $\mathscr{Q} \in \theta X \backslash E X$, then $\left\{\pi_{\kappa}(\mathscr{C})\right\}=$ $\operatorname{ad}_{\kappa X} \mathscr{U}$ implying $\pi_{\kappa}(\mathscr{U})=\mathscr{W}$ and $\left(f \circ \pi_{\kappa}\right)(\mathscr{U})=\pi_{Y}(\mathscr{U})$. So, we have that $f \circ \pi_{x}=\pi_{Y}$. Suppose $Z$ is an $H$-closed extension of $X$ and $g: \kappa X \rightarrow Z$ is the unique continuous function that extends the identity function on $X$. Then we have $g \circ \pi_{k}=\pi_{z}$. Also, suppose that $h: Y \rightarrow Z$ is a continuous function that extends the identity function on $X$. By the uniqueness of the extension function $g$, it follows that $h \circ f=g$. Thus, $h \circ \pi_{Y}=h \circ f \circ \pi_{k}=g \circ \pi_{\kappa}=\pi_{Z}$.

An immediate consequence of this fact and 3.4 is the next result.

Proposition 3.5. Let $Y$ and $Z$ be $H$-closed extensions of a space $X$ and suppose there is a continuous function $h: Y \rightarrow Z$ that extends the identity function on $X$. Suppose $X \subseteq T \subseteq Z$. Then $T$ and $h^{-}(T)$ are co-absolute.

Throughout this section $\mathscr{P}$ will denote a class of spaces with these properties:

(i) $\mathscr{P}$ contains the class of $H$-closed spaces.

(ii) $\mathscr{P}$ is co-absolute closed, i.e., if $X \in \mathscr{P}, Y$ is co-absolute with $X$, then $Y \in \mathscr{P}$.

(iii) $\mathscr{P}$ has the $\kappa$-intersection property.

These three requirements on $\mathscr{P}$ guarantee that $\mathscr{P}$ is an extension property (as defined in 2.1) and has other nice properties as proved in the next result.

Proposition 3.6. (a) Let $Y$ be an extension of a space $X$ and $\mathscr{F}$ be a nonempty family of $\mathscr{P}$-extensions $Z$ such that $X \subseteq Z \subseteq Y$. Then $\cap \mathscr{F}$ is a $\mathscr{P}$-extension of $X$.

(b) $\mathscr{P}$ has the $\kappa$-inversion property.

(c) $\mathscr{P}$ is an extension property.

Proof. To prove (a), let $h Y$ be an $H$-closed extension of $Y$ and $f: \kappa X \rightarrow h Y$ the unique continuous function that extends the identity function on $X$ (see 1.9). By 3.5, for $Z \in \mathscr{F}, Z$ and $f^{\leftarrow}(Z)$ are coabsolute. So, $f^{\leftarrow}(Z) \in \mathscr{P}$. Thus, $T=\bigcap\left\{f^{\leftarrow}(Z): Z \in \mathscr{F}\right\} \in \mathscr{P}$. Let $S=\bigcap\{Z: Z \in \mathscr{F}\}$. Then $T=f^{\leftarrow}(S)$ and by 3.5, $T$ and $S$ are coabsolute. So, $S \in \mathscr{P}$. Now (b) follows from 3.5 and (c) follows from (b) and 2.5.

Definition 3.7. Let $Y$ be a $\mathscr{P}$-extension of $X . \quad Y$ is called a tight $\mathscr{P}$-extension if whenever $Z$ is a $\mathscr{P}$-extension of $X$ and 
$X \subseteq Z \subseteq Y$, it follows that $Z=Y$.

By 3.6, for each $H$-closed extension $h X$ of a space $X$, there is a tight $\mathscr{P}$-extension, denoted by $h_{\mathscr{S}} X$, such that $X \subseteq h_{\mathscr{S}} X \subseteq h X$. By 2.2 , this notation for the $H$-closed extension $\kappa X$ conforms with the notation introduced in 2.1. If $a X$ is a tight $\mathscr{P}$-extension of $X$, then the Katetov extension $\kappa a X$ of $a X$ is an $H$-closed extension of $X$; it follows that $(\kappa a), X=a X$. Thus, $\left\{h_{,} X: h X \in \mathscr{C}(X)\right\}$ is the set of all tight $\mathscr{P}$-extensions of $X$ and is denoted by $\mathscr{P}(X)$. By 2.1 and 2.2 , it follows that $\kappa_{\mathscr{O}} X \geqq Y$ for all $Y \in \mathscr{P}(X)$.

TheOREm 3.8. Let $X$ be a space and $Y, Z \in \mathscr{P}(X)$. Then $Y \geqq Z$ iff $\kappa Y \geqq \kappa Z$.

Proof. Suppose $Y \geqq Z$. There is a continuous function $f: Y \rightarrow Z$ such that $f(x)=x$ for all $x \in X$. To show $f$ extends to a continuous function from $\kappa Y$ to $\kappa Z$, it suffices by [5] to show that $f$ is a $p$-map, i.e., for each $p$-cover $\mathscr{C}$ of $Z$ (i.e., $\mathscr{C}$ is an open cover with a finite subfamily whose union is dense), $f^{\leftarrow}(\mathscr{U})=\left\{f^{\leftarrow}(U): U \in \mathscr{C}\right\}$ is a $p$-cover of $Y$. Now, $f^{\leftarrow}(\mathscr{Q})$ is an open cover of $Y$. Let $U_{1}, \cdots, U_{n}$ be a subfamily of $\mathscr{C}$ whose union is dense in $Z$. Then $\bigcup\left\{U_{i} \cap X: 1 \leqq\right.$ $i \leqq n\}$ is open and dense in $X$. Sinse $\bigcup\left\{U_{i} \cap X: 1 \leqq i \leqq n\right\} \leqq$ $\bigcup\left\{f^{\leftarrow}\left(U_{i}\right): 1 \leqq i \leqq n\right\}$, then $f^{\leftarrow}\left(U_{i}\right): 1 \leqq i \leqq n$ is a subfamily whose union is dense in $Y$. Thus, $f^{\leftarrow}(\mathscr{Q})$ is a $p$-cover. Conversely, suppose $\kappa Y \geqq \kappa Z$. Let $F: \kappa Y \rightarrow \kappa Z$ be a continuous function that extends the identity function on $X$. By 3.5, $Z$ and $F^{\leftarrow}(Z)$ are co-absolute; since $Z \in \mathscr{P}$, then $F^{\leftarrow}(Z) \in \mathscr{P}$. Since $Y$ is a tight $\mathscr{P}$-extension, then $Y \subseteq F[Z]$. Thus, $F \mid Y: Y \rightarrow Z$ and $Y \geqq Z$.

Let $Y$ be an extension of $X$ and $f: \kappa X \rightarrow \kappa Y$ be the unique continuous extension of the identity function on $X$ (see 1.9). Let $P(Y)=\left\{f^{\leftarrow}(p): p \in Y \backslash X\right\} ; P(Y)$ is a partition of $f^{\leftarrow}(Y \backslash X)$, which is contained in $\kappa X \backslash X$ since $f \mid X=1_{X}$. Since $\sigma X$ and $\kappa X$ have the same underlying set, then $f^{-}(Y \backslash X) \leqq \sigma X \backslash X$, and by (0.5) in [13], $P(Y)$ is a collection of pairwise disjoint compact subspaces of $\sigma X \backslash X$. If $Y$ and $Z$ are extensions of $X$, then $P(Y)$ refines $P(Z)$ if for each $A \in P(Y)$, there is a $B \in P(Z)$ such that $A \subseteq B$; in particular, $\mathrm{U} P(Y) \subseteq \mathrm{U} P(Z)$.

Lemma 3.9. If $Y$ and $Z$ are extensions of $X$ such that $Y \geqq Z$, then $P(Y)$ refines $P(Z)$.

Proof. Let $f: \kappa X \rightarrow \kappa Y$ and $g: \kappa X \rightarrow \kappa Z$ be continuous extensions of the identity function on $X$. By 3.8 , there is a continuous function $h: \kappa Y \rightarrow \kappa Z$ such that $h(Y) \subseteq Z$ and $h$ extends the identity function 
on $X$. By the uniqueness of $g, h \circ f=g$. For $p \in Y \backslash X, f^{\leftarrow}(p) \in P(Y)$ and $f^{\leftarrow}(p) \subseteq f^{\leftarrow}\left(h^{\leftarrow}(h(p))\right)=g^{\leftarrow}(h(p)) \in P(Z)$. So, $P(Y)$ refines $P(Z)$.

Let $Y$ be an extension of $X$ and $f: \kappa X \rightarrow \kappa Y$ be the continuous extension of the identity function on $X$. For $p \in \kappa Y$, let $O(p)$ be the open neighborhood filter trace of $p$ on $X$. In particular, if $\mathscr{C} \in \kappa X \backslash X$, then $f(\mathscr{C})$ is the unique point in $\kappa Y \backslash X$ such that $\mathscr{U} \supset O(f(\mathscr{W})$. (See paragraph after 3.4.) Also, if $\mathscr{W} \in \kappa Y \backslash Y$, let $\mathscr{W}_{X}=\{W \cap X: W \in \mathscr{W}\} \in \kappa X \backslash X$; thus, $f^{\leftarrow}(\mathscr{W})=\left\{\mathscr{W}_{x}\right\}$. If $\mathscr{T}$ is an open filter on $X$, let $\mathscr{F}_{S}$ denote the open filter generated by the open filter base $\left\{\operatorname{int}_{x}\left(\mathrm{cl}_{X} F\right): F \in \mathscr{F}\right\}$. It is easy to show that if $\mathscr{C}$ is an open ultrafilter and $\mathscr{F}$ is open filter on $X$, then $\mathscr{U} \supseteq \mathscr{F}$ iff $\mathscr{U} \supseteqq \mathscr{F}_{S}$.

Let $Y$ and $Z$ be extensions of a space $X$. The extensions $Y$ and $Z$ are said to be $\theta$-isomorphic if there is a $\theta$-homeomorphism (i.e., a bijection that is $\theta$-continuous (see 3.1) in both directions) between $Y$ and $Z$ that extends the identity function on $X$. The proof of the following lemma is straightforward and left to the reader.

LEMMA 3.10. Let $Y$ and $Z$ be extensions of a space $X$, and let $f: Y \rightarrow Z$ be a $\theta$-continuous function such that $f(x)=x$ for all $x \in X$. Let $p \in Y$. Then $(O(f(p)))_{s} \subseteq(O(p))_{S}$.

Theorem 3.11. Let $Y, Z \in \mathscr{P}(X)$ for a space $X$. Consider the following:

(a) $Y$ and $Z$ are $\theta$-isomorphic.

(b) $P(Y)=P(Z)$.

(c) $\kappa Y$ and $\kappa Z$ are $\theta$-isomorphic.

Then (a) is equivalent to (b) and implies (c).

Proof. Let $f: \kappa X \rightarrow \kappa Y$ and $g: \kappa X \rightarrow \kappa Z$ be the continuous functions that extend the identity function on $X$.

(a) implies (b). Let $h: Y \rightarrow Z$ be the $\theta$-homeomorphism that extends the identity function on $X$. If $p \in Y$, by $3.10,(O(p))_{s}=$ $(O(h(p)))_{s}$. Thus, if $p \in Y \backslash X, \mathscr{C} \in f^{\leftarrow}(p)$ iff $\mathscr{Q} \supseteqq O(p)$. But $\mathscr{C} \supseteqq$ $(O(p))_{S}$ iff $\mathscr{C} \in g^{\leftarrow}(h(p))$. So, $f^{\leftarrow}(p)=g^{\leftarrow}(h(p))$ and $P(Y)=P(Z)$.

(b) implies (a) and (c): Suppose $P(Y)=P(Z)$. Recall that $f^{\leftarrow}(\mathscr{C})=\left\{\mathscr{U}_{X}\right\}$ for $\mathscr{C} \in \kappa Y \backslash Y$. Then $P(\kappa Y)=\left\{f^{\leftarrow}(p): p \in \kappa Y \backslash X\right\}=$ $P(Y) \cup\left\{\left\{\mathscr{U}_{X}\right\}: \mathscr{W} \in \kappa Y \backslash Y\right\}=P(Y) \cup\left\{\{\mathscr{V}\}: \mathscr{V} \in \kappa X \backslash f^{\leftarrow}(Y)\right\}=P(Z) \cup\{\{\mathscr{V}\}:$ $\left.\mathscr{V} \in \kappa X \backslash g^{-}(Z)\right\}=P(Z) \cup\left\{\left\{\mathscr{U}_{X}\right\}: \mathscr{U} \in \kappa Z \backslash Z\right\}=\left\{g^{-}(p): p \in \kappa Z \backslash X\right\}=P(\kappa Z)$. The quotient topology on the partition $P(\kappa Y) \cup\{\{x\}: x \in X\}$ of $X$ is an $H$-closed extension $h X$ of $X$ (see Theorem 2.1 in [15]) and $h X$ is $\theta$-isomorphic to $\kappa Y$ and to $\kappa Z$. Let $k: \kappa X \rightarrow h X$ be the continuous 
extension of the identity function on $X$. There are continuous bijections $r: h X \rightarrow \kappa Y$ and $s: h X \rightarrow \kappa Z$ that leave $X$ pointwise fixed and both $r^{\leftarrow}$ and $s^{-}$are $\theta$-continuous. Now, by uniqueness, $r \circ k=f$ and $s \circ k=g$. Also, $s \circ r^{\leftarrow}: \kappa Y \rightarrow \kappa Z$ is a $\theta$-isomorphism. If $p \in Y \backslash X$, then $g^{\leftarrow}\left(s^{\circ} \circ(p)\right)=\left(k^{\leftarrow} \circ s^{\leftarrow}\right)\left(s^{\leftarrow} \circ r^{\leftarrow}(p)\right)=k^{\leftarrow} \circ r^{\leftarrow}(p)=f^{\leftarrow}(p)$. But $f^{\leftarrow}(p) \in P(Y)$ implies $g^{\leftarrow}\left(\operatorname{s}^{\circ}(p)\right) \in P(Z)$. So, $s^{\circ} r^{\leftarrow}(p) \in Z \backslash X$ and $s \circ r^{\leftarrow}(Y) \subseteq Z$. Similarly, $r \circ s^{\leftarrow}(Z) \subseteq Y$. Thus, $s \circ r^{\leftarrow} \mid Y: Y \rightarrow Z$ is a bijection that extends the identity function on $X$. By 3.3, $s \circ r^{\leftarrow} \mid Y$ is a $\theta$-homeomorphism.

The property of $\theta$-isomorphism is an equivalence relation in $\mathscr{P}(X)$; let $A$ be an element of the induced partition on $\mathscr{P}(X)$ and $Y \in A$. Then $P(\kappa Y)$ is partition of $\kappa X \backslash X$ and the induced quotient space $h X$ is $H$-closed and $h X \geqq \kappa Y$. In fact, for $Z \in A, h X \geqq \kappa Z$ and the continuous function $f: h X \rightarrow \kappa Z$ that leaves $X$ pointwise fixed is a $\theta$-isomorphism (the proofs of these two fact are similar to the proofs of the corresponding results in [15] and [16]). By 3.5, $Z$ and $f^{\leftarrow}(Z)$ are co-absolute; since $Z \in \mathscr{P}$, then $f^{\leftarrow}(Z) \in \mathscr{P}$. Thus, $h_{\mathscr{O}} X \subseteq f^{\leftarrow}(Z)$ and $h_{\mathscr{S}} X \geqq Z$ for all $Z \in A$. So, $h_{\Im} X$ is a projective maximum of $A$. Let $\mathscr{P}^{\theta}(X)$ denote the set of projective maxima, one from each $\theta$-isomorphism class in $\mathscr{P}(X)$.

Lemma 3.12. Let $Y$ and $Z \in \mathscr{P}^{\theta}(X)$. Then $Y \geqq Z$ iff $P(Y)$ refines $P(Z)$.

Proof. By 3.9, if $Y \geqq Z$, then $P(Y)$ refines $P(Z)$. Conversely, suppose $P(Y)$ refines $P(Z)$. Then, $P(\kappa Y)$ refines $P(\kappa Z)$. Now, $P(\kappa Y)$ induces an $H$-closed extension $h_{Y} X$ of $X$ and the tight $\mathscr{P}$-extension of $h_{Y} X$ is $Y$. By (0.6) in [13], $h_{Y} X \geqq h_{Z} X$. Let $f: h_{Y} X \rightarrow h_{Z} X$ be the continuous extension of the identity function on $X$. By 3.5, $Z$ and $f^{\leftarrow}(Z)$ are co-absolute. So, since $Z$ belongs to $\mathscr{P}$, then so does $f^{\leftarrow}(Z)$. Thus, $Y \subseteq f^{\leftarrow}(Z)$ as $Y$ is tight. This shows that $Y \geqq Z$.

For a space $X$, let $\sigma_{\mathscr{S}} X$ denote the tight $\mathscr{P}$-extension of $X$ induced by $\sigma X$ (see 1.8(a)). As noted in the paragraph following $3.7, \kappa_{\mathscr{S}} X \geqq \sigma_{\mathscr{S}} X$. Let $f: \kappa X \rightarrow \sigma X$ be the continuous extension of the identity function on $X ; f$ is a bijection and is the identity function on the common underlying set of $\kappa X$ and $\sigma X$. So, $f\left(\kappa_{\mathscr{S}} X\right) \subseteq$ $\sigma_{\mathscr{S}} X$. Since, by 3.5, $f\left(\kappa_{\mathscr{O}} X\right)$ and $f^{\leftarrow} f\left(\kappa_{\mathscr{O}} X\right)=\kappa_{\mathscr{S}} X$ are co-absolute, then $f\left(\kappa_{\mathscr{P}} X\right)$ is a $\mathscr{P}$-extension of $X$. Thus $\sigma_{\mathscr{P}} X=f\left(\kappa_{\mathscr{S}} X\right)$. So, as sets, $\sigma_{\mathscr{P}} X=\kappa_{\mathscr{P}} X$. If $Y \in \mathscr{P}(X)$ and $g: \kappa X \rightarrow \kappa Y$ is the continuous extension of the identity function on $X$, then $g\left(\kappa_{g} X\right) \subseteq Y$. Thus, $\mathrm{U} P(Y)=g^{\leftarrow}(Y \backslash X) \supseteqq \kappa_{\mathscr{P}} X \backslash X . \quad$ Let $\mathscr{D}(X)=\left\{Y \in \mathscr{P}^{\theta}(X): \kappa_{\mathscr{O}} X \backslash X=\right.$ $\mathrm{U} P(Y)\}$. Hence, for $Y \in \mathscr{D}(X), P(Y)$ is a partition of $\kappa_{\mathscr{O}} X \backslash X$ and of $\sigma_{\mathscr{P}} X \backslash X$. 
LEMMa 3.13. Let $X$ be a space and $P$ a partition of $\sigma_{\mathscr{P}} X \backslash X$. Then $P=P(Y)$ for some $Y \in \mathscr{D}(X)$ iff the elements of $P$ are compact subspaces of $\sigma X$.

Proof. If $P=P(Y)$ for some extension $Y$ of $X$, then the elements of $P$ are compact subspaces of $\sigma X$ (see Cor. 5.5 in [16]). Conversely, suppose the elements of $P$ are compact subspaces of $\sigma X$. Then $P \cup\{\{\mathscr{C}\}: \mathscr{Q} \in \sigma X \backslash[X \cup(\cup P)]\} \cup\{\{x\}: x \in X\}$ is a partition of compact subsets of $\sigma X$; the quotient space $h X$ of $\kappa X$ induced by this partition is an $H$-closed extension of $X$ (see Cor. 5.5 in [16]). Let $f: \kappa X \rightarrow h X$ be the quotient map; thus $f$ is a continuous extension of the identity function on $X$. Let $Z=f \mid \cup P] \cup X$; clearly $P(Z)=P$. Since $\bigcup P(Z)=\bigcup P=\kappa_{3} X \backslash X$, then $f^{\leftarrow}(Z)=\kappa_{\mathscr{O}} X$. Since, by $3.5, Z$ and $f^{\leftarrow}(Z)=\kappa, X$ are co-absolute, then $Z$ is a $\mathscr{P}$-extension of $X$. By the paragraph following $3.11, h_{\mathscr{O}} X \in \mathscr{P}^{\theta}(X)$. So, it suffices to show $Z=h_{\mathscr{\Im}} X$. Since $h_{\Im} X$ is tight, then $Z \supseteq h_{9} X$. Since $\kappa_{\mathscr{P}} X \geqq h_{\mathscr{O}} X$, then $Z=f\left(\kappa_{\mathscr{P}} X\right) \subseteq h_{\mathscr{P}} X$. So, $Z=h_{\Im} X, h_{\mathscr{S}} X \in \mathscr{D}(X)$, and $P=P\left(h_{\Im} X\right)$.

Let $X$ be a space, and let $\mathscr{L}_{\mathscr{P}}=\left\{P: P\right.$ is a partition of $\sigma_{\mathscr{O}} X \backslash X$ whose members are compact subspaces\}. Then $\mathscr{L}_{\mathscr{G}}$ is partially ordered by refinement. Recall that $\mathscr{D}(X)$ is partially ordered by $\leqq$. The following corollary is an easy consequence of $3.11,3.12$, 3.13 and the definition of $\mathscr{P}^{\theta}(X)$.

CoROLlaRY 3.14. Let $X$ be a space. The function $\phi: \mathscr{D}[X] \rightarrow \mathscr{L}_{\mathscr{G}}$ defined by $\phi(Y)=P(Y)$ is an order-preserving isomorphism.

Note that for a space $X$ and $X \subseteq T \subseteq \sigma X$, it is not necessarily true that $\sigma T$ and $\sigma X$ are isomorphic extensions of $X$, see Example 7.7 in [16]. However, we have the next result.

THEOREM 3.15. Let $X$ be a space and $X \subseteq T \subseteq \sigma X$. Then $\sigma X \backslash T$ and $\sigma T \backslash T$ are homeomorphic.

Proof. Let $\mathscr{U}_{C}$ be a free open ultrafilter on $T$. Then $\mathscr{C}_{x}$ $(=\{U \cap X: U \in \mathscr{U}\})$ is a free open ultrafilter on $X$ and $\mathscr{U}_{X} \in T$. Thus we can define a function $g: \sigma T \backslash T \rightarrow \sigma X \backslash T$ by $g(\mathscr{C})=\mathscr{U}_{x}$. If $\mathscr{U}, \mathscr{V} \in \sigma T \backslash T$ and $\mathscr{U} \neq \mathscr{V}$, there are disjoint open sets $U \in \mathscr{U}$ and $V \in \mathscr{V}$. Since $U \cap X$ and $V \cap X$ are disjoint, then $\mathscr{C}_{X} \neq \mathscr{V}_{X}$ and $g$ is one-to-one. If $\mathscr{W} \in \sigma X \backslash T$, then since $X$ is dense, it follows that $\mathscr{W}=\{U: U$ open in $T$ and $U \cap X \in \mathscr{W}\}$ is a free open ultrafilter on $T$ and $\mathscr{U}_{X}=\mathscr{W}$. Thus, $g$ is a bijection. Let $U$ be an open set in $X$ and $O_{U}^{\prime}(=\{\mathscr{U} \in \sigma X \backslash X: U \in \mathscr{C}\} \cup U)$ a basic open set in $\sigma X$ as 
defined in 1.8. Let $W=O_{U}^{\prime} \cap T$ and let $O_{W}^{\prime}$ be the corresponding basic open set in $\sigma T$. To show the bijection $g$ is a homeomorphism, it suffices to show that $g\left(O_{W} \backslash T\right)=O_{U} \backslash T$. Note that $W \cap X=U$. For $\mathscr{W} \in \sigma T \backslash T, \mathscr{W} \in O_{W} \backslash T$ iff $W \in \mathscr{W}$, i.e., $W \cap X \in \mathscr{W}_{X}$. But $U=W \cap X \in \mathscr{W}_{X}$ is equivalent to $g\left(\mathscr{W}^{-}\right)=\mathscr{W}_{X} \in O_{U} \backslash T$.

For a space $X$, let $\delta X=X \cup\left(\sigma X \backslash \sigma_{\mathscr{S}} X\right)$. Then $X \subseteq \delta X \leqq \sigma X$ and $\sigma X \backslash \delta X=\sigma_{\sigma} X \backslash X$. Let $\mathscr{L}_{\delta}=\{P: P$ is a partition of $\sigma(\delta X) \backslash \delta X$ into compact subspaces\}. $\mathscr{L}_{\delta}$ is partially ordered by refinement. By $3.15, \sigma X \backslash \delta X$ and $\sigma(\delta X) \backslash \delta X$ are homeomorphic and, hence, the next result follows:

Corollary 3.16. Let $X$ be a space. Then $\mathscr{L}_{\infty}$ and $\mathscr{L}_{0}$ are order-isomorphic.

In the particular case when $\mathscr{P}$ is the class of all $H$-closed extensions, $\mathscr{P}$ is co-absolute closed and has the $\kappa$-intersection property (see the paragraph following 3.5). The set of tight $\mathscr{P}$-extensions is the set of all $H$-closed extensions; for a space $X, h, X=h X$ for each $H$-closed extension $h X$ of $X$ and $\mathscr{P}^{\theta}(X)=\mathscr{D}(X)$. In $[P]$, $\mathscr{P}^{\theta}(X)$ is denoted by $\mathscr{H}^{\theta}(X)$. The next result relates $\mathscr{D}(X)$ for an arbitrary $\mathscr{P}$ (that is co-absolute closed and has $\kappa$-intersection property) with its $H$-closed extension structure $\mathscr{H}^{\theta}(X)$.

Theorem 3.17. Let $X$ be a space. Then $\mathscr{D}(X)$ and $\mathscr{H}^{\theta}(\delta X)$ are order-isomorphic.

Proof. By 3.14 and 3.16 , it suffices to show that $\mathscr{L}_{i}$ and $\mathscr{H}^{\theta}(\delta X)$ are order-isomorphic which follows immediately from (0.5) and (0.6) in [13].

For a space $X$, let $k X$ denote the $k$-space co-reflection of $X$, i.e., $X$ with $\{A \subseteq X: A \cap C$ closed for every compact $C \cong X\}$ as a topology for the closed sets.

Theorem 3.18. Let $X$ and $Y$ be spaces. Then $\mathscr{D}(X)$ and $\mathscr{D}(Y)$ are order-isomorphic iff $k\left(\sigma_{\mathscr{S}} X \backslash X\right)$ and $k\left(\sigma_{\mathscr{D}} Y \backslash Y\right)$ are homeomorphic.

Proof. By 3.17, $\mathscr{D}(X)$ and $\mathscr{D}(Y)$ are order-isomorphic iff $\mathscr{H}^{\theta}(\delta X)$ and $\mathscr{C}^{\theta}(\delta Y)$ are order-isomorphic which is equivalent to $\mathscr{H}^{\theta}(\delta X)$ and $\mathscr{H}^{\theta}(\delta Y)$ being lattice isomorphic. By Theorem 2.3 in [13], $\mathscr{H}^{\theta}(\delta X)$ and $\mathscr{H}^{\theta}(\delta Y)$ are lattice isomorphic iff $k(\sigma(\delta X) \backslash \delta X)$ and $k(\sigma(\delta Y) \backslash \delta Y)$ are homeomorphic. By 3.15 and the paragraph following 
3.15, this latter statement is equivalent to $k\left(\sigma_{\mathscr{S}} X \backslash X\right)$ and $k\left(\sigma_{\mathscr{S}} Y \backslash Y\right)$ being homeomorphic.

When $\mathscr{P}$ is the class of all $H$-closed spaces, then for each space $X, \mathscr{H}^{\theta}(X)=\mathscr{D}(X)$ and $\sigma_{\mathscr{O}} X=\sigma X$; the result in Theorem 3.18 is precisely the same result as in Theorem 2.3 in [13]. However, Theorem 3.18 is a pure extension of Theorem 2.3 in [13] when $\mathscr{P}$ is the class of all almost realcompact spaces. This particular $\mathscr{P}$ is co-absolute closed and has the $\kappa$-intersection property (see 2.15), and the maximal almost realcompact extension $\kappa_{\mathscr{9}} X$ of a space $X$ is denoted as $\rho X$ by Liu and Strecker in [10].

Let $m$ be an infinite cardinal and $\mathscr{R}$ the class of all $m$-H-closed spaces, as defined in 2.19. It is straightforward to show for a space $X$ such that $E X \in \mathscr{R}$, then $X \in \mathscr{R}$. Using 3.2, it easily follows that $\hat{\mathscr{R}}$ is co-absolute closed, and by $2.11, \hat{\mathscr{R}}$ has the $\kappa$-intersection property. Thus, for each infinite cardinal $m, \hat{\mathscr{R}}$ is another class of Hausdorff spaces satisfying 3.18.

For the class $\mathscr{P}$ of all almost realcompact spaces, let $\mathscr{P}^{\prime}$ be the class of all $\mathscr{P}$-pseudo- $H$-closed spaces defined in 2.7. In the next result, $\mathscr{P}^{\prime}$ is proven to be exactly the class of all $\aleph_{0}-H$-closed spaces (sometimes called feebly compact spaces, see 2.19). Thus, by the preceding paragraph, $\widehat{\mathscr{\mathscr { P }}^{\prime}}$ satisfies 3.18 . By $2.12 \mathscr{P}_{\widehat{P}}^{\mathscr{P}^{\prime}}$.

THEOREM 3.19. Let $X$ be a space and $\mathscr{P}$ the class of all almost realcompact spaces. Then $X \in \mathscr{P}^{\prime}$ iff $X$ is $\aleph_{0}$-H-closed.

Proof. Suppose $X \in \mathscr{P}^{\prime}$ and assume there is a countable open cover $\mathscr{C}$ of $X$ without a finite subfamily whose union is dense. Without loss of generality suppose $\mathscr{C}$ is closed under finite unions. Now $\{X \mid$ cl $U: U \in \mathscr{C}\}$ is contained in a free open ultrafilter $\mathscr{C} \in \kappa X \backslash X$. Since $X \in \mathscr{P}^{\prime}$, then by $2.7, \kappa_{,} X=\kappa X$. So, $\mathscr{\mathscr { C }} \in \kappa_{\mathscr{S}} X \backslash X$ and by $2.3(\mathrm{~b})$, $\varnothing \neq \bigcap\{\operatorname{cl}(X \mid \operatorname{cl} U): U \in \mathscr{C}\}$. Since $\operatorname{cl}(X \mid \operatorname{cl} U) \subseteq X \backslash U$ and $\cap\{X \backslash U$ : $U \in \mathscr{C}\}=\varnothing$, then $\bigcap\{\operatorname{cl}(X \mid \operatorname{cl} U): U \in \mathscr{C}\}=\varnothing$, a contradiction. Conversely, suppose $X$ is $\boldsymbol{W}_{0}-H$-closed. Let $\mathscr{C}$ be a free open ultrafilter on $X$ and $\left\{U_{n}: n \in N\right\} \subseteq \mathscr{U}$. Now, $\bigcap\left\{\right.$ cl $\left.U_{n}: n \in N\right\} \neq \varnothing$ or else $\left\{X \backslash \mathrm{cl} U_{n}: n \in N\right\}$ is open cover of $X$ and there is $m \in N$ such that $\varnothing=\bigcap\left\{\operatorname{cl} U_{i}: 1 \leqq i \leqq m\right\}$ contradicting the fact that $\mathscr{W}$ is a filter.

REMARK 3.20. In this section we have obtained a Magill-like result (Theorem 3.18) for an extension property which is co-absolutely closed. Also, 3.18 is true for an extension property satisfying this condition. 
(ii) $^{\prime}$ If $Y$ is an extension of $X$ and $f: \kappa X \rightarrow \kappa Y$ is the continuous extension of the identity function on $X$, then $Y \in \mathscr{P}$ iff $f^{\leftarrow}(Y) \in \mathscr{P}$.

By 3.5, a co-absolutely closed property satisfies (ii)', and it is straightforward to show that a property $\mathscr{P}$ satisfying (ii)' also satisfies the following modification of 3.5 :

Proposition 3.5'. Let $Y$ and $Z$ be $H$-closed extensions of a space $X$ and suppose there is a continuous function $h: Y \rightarrow Z$ that extends the identity function on $X$. If $X \subseteq T \leqq Z$, then $T \in \mathscr{P}$ iff $h^{\leftarrow}(T) \in \mathscr{P}$.

Now, if 3.5 is replaced by $3.5^{\prime}$ in the proofs, then 3.6 through 3.18 are true for an extension property satisfying (ii)'.

\section{REFERENCES}

1. W. W. Comfort and S. Negrepontis, The Theory of Ultrafilters, Springer-Verlag, New York, 1974.

2. R. F. Dickman Jr, and J. R. Porter, $\theta$-perfect and $\theta$-absolutely continuous functions, Illinois J. Math., 21 (1977), 42-60.

3. Z. Frolik, A generalization of realcompact spaces, Czech. Math. J., 13 (1963), 127-138.

4. L. Gillman and M. Jerison, Rings of Continuous Functions, Van Nostrand, New York, 1960.

5. D. Harris, The Katetov extension as a functor, Math. Ann., 193 (1971), 171-175.

6. H. Herrlich and J. van der Slot, Properties which are closely related to compactness, Indag. Math., 29 (1967), 524-529.

7. S. Iliadis and S. Fomin, The method of centered systems in the theory of topological spaces, Uspehi Mat. Nauk., 21 (1966), 47-76; (in Russian; English Transl: Russian Math. Surveys, 21 (1966), 37-62).

8. I. Juhasz, Cardinal functions in topology, Math. Centre Tracts No. 34 (Mathematisch Centrum, Amsterdam, 1971).

9. M. Katetov, Über H-abgeschlossen und bikompakt Raüme, Casopis pro. Mat. Fys., 69 (1940), 36-49.

10. C. T. Liu and G. E. Strecker, Concerning almost realcompactifications, Czech. Math. J., 22 (1972), 181-190.

11. K. D. Magill Jr., The lattice of compactifications of a locally compact space, Proc. London Math. Soc., (3) 18 (1968), 231-244.

12. J. Mack, M. Rayburn and R. G. Woods, Lattices of topological extensions, Trans. Amer. Math. Soc., 189 (1974), 163-174.

13. J. R. Porter, Lattices of H-closed extensions, Bull. Acad. Polon. Sci. Ser. Math. Astr. Phys., 22 (1974), 831-837.

14. J. R. Porter and J. D. Thomas, On H-closed and minimal Hausdorff spaces, Trans. Amer. Math. Soc., 138 (1969), 159-170.

15. J. R. Porter and C. Votaw, H-closed extensions I, General Topology and Appl., 3 (1973), 211-224.

16. - H-closed extensions II, Trans. Amer. Math. Soc., 202 (1975), 193-209.

17. M. E. Rudin, Lectures in set-theoretic topology, C.B.M.S. Regional Conference series in Mathematics No. 23, (1974).

18. M. Ulmer, Products of weakly 3 -compact spaces, Trans. Amer. Math. Soc., 170 (1972), 279-284.

19. S. Willard, General Topology, Addison-Wesley, Reading, Mass., 1970. 
20. R. G. Woods, Topological extension properties, Trans. Amer. Math. Soc., 210 (1975), 365-385.

21. - Characterizations of some $C^{*}$-embedded subspaces of $\beta N$, Pacific J. Math., 65 (1976), 573-579.

22. R. G. Woods, Epireflective subcategories of Hausdorff categories, to appear in Topology and its Applications.

Received May 29, 1981. The research of the first author was partially supported by the University of Kansas General Research Fund. The research of the second author was partially supported by a grant (No. A 7592) from the National Research Council of Canada.

The UNIVERSITY OF Kansas

LAWRENCE, KS

AND

The University of Manitoba

Winnipeg, Manitoba, Canada 


\section{PACIFIC JOURNAL OF MATHEMATICS}

\section{EDITORS}

DONALD BABBITT (Managing Editor)

University of California

Los Angeles, California 90024

Hugo RossI

University of Utah

Salt Lake City, UT 84112

C. C. Moore and Arthur Agus

University of California

Berkeley, CA 94720
J. DugundJI

Department of Mathematics University of Southern California Los Angeles, California 90007

R. FInN and J. Milgram Stanford University Stanford, California 94305

ASSOCIATE EDITORS
R. ARNES
E. F. BeCKenbach
B. H. NeumanN
F. WOLF
K. YoSHIDA

\section{SUPPORTING INSTITUTIONS}

UNIVERSITY OF ARIZONA

UNIVERSITY OF BRITISH COLUMBIA

CALIFORNIA INSTITUTE OF TECHNOLOGY

UNIVERSITY OF CALIFORNIA

MONTANA STATE UNIVERSITY

UNIVERSITY OF NEVADA, RENO

NEW MEXICO STATE UNIVERSITY

OREGON STATE UNIVERSITY
UNIVERSITY OF OREGON

UNIVERSITY OF SOUTHERN CALIFORNIA

STANFORD UNIVERSITY

UNIVERSITY OF HAWAII

UNIVERSITY OF TOKYO

UNIVERSITY OF UTAH

WASHINGTON STATE UNIVERSITY

UNIVERSITY OF WASHINGTON 


\section{Pacific Journal of Mathematics}

\section{Vol. 103, No. $1 \quad$ March, 1982}

Abdul Aziz, On the zeros of composite polynomials ..................

Salomon Benzaquen and Enrique M. Cabaña, The expected measure of the level sets of a regular stationary Gaussian process $\ldots \ldots \ldots \ldots \ldots$

Claudio D’Antoni, Roberto Longo and László Zsidó, A spectral mapping theorem for locally compact groups of operators $\ldots \ldots \ldots \ldots \ldots \ldots \ldots 17$

Ronald Dotzel, Semifree finite group actions on homotopy spheres ........ 25

Daniel H. Gottlieb, The Lefschetz number and Borsuk-Ulam theorems . . . . . 29

Shui-Hung Hou, On property $(Q)$ and other semicontinuity properties of

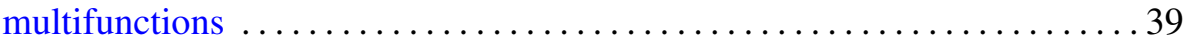

Kevin Mor McCrimmon, Compatible Peirce decompositions of Jordan triple systems

Mitsuru Nakai, Corona problem for Riemann surfaces of Parreau-Widom

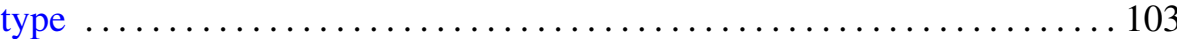

Jack Ray Porter and R. Grant Woods, Extensions of Hausdorff spaces . . . . 111

Milton Rosenberg, Quasi-isometric dilations of operator-valued measures

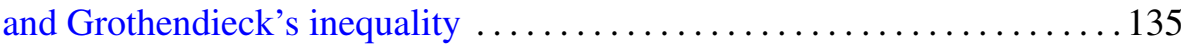

Joseph L. Taylor, A bigger Brauer group $\ldots \ldots \ldots \ldots \ldots \ldots \ldots \ldots \ldots \ldots$

Thomas Vogel, Symmetric unbounded liquid bridges . . . . . . . . . . . 205

Steve Wright, The splitting of operator algebras. II ............... 243 